Gail M. Thornton

University of Alberta, Edmonton, Alberta, Canada

Emily Kroeker

University of Alberta, Edmonton, Alberta, Canada

\title{
Overlay Journals: Overlooked or Emergent? (Paper)
}

\begin{abstract}
Résumé:
Overlay journals are not a new concept but have been experiencing a recent resurgence because of the increase in the number of preprint servers and the increase in the number of preprints on coronavirus disease 2019 (COVID-19) related topics. This study examines overlay journals at various stages of maturity for unique characteristics, including whether the authors submitted their article to the journal and whether the reviews of the article are published by the overlay journal. Whether they are considered overlooked or emergent, overlay journals are becoming an important contribution to scholarly communication.
\end{abstract}

\section{Introduction}

The term overlay journal was coined in 1996 by Paul Ginsparg, who developed the arXiv preprint server in 1991 (Brown 2010). An overlay journal is "[a]n open-access journal that takes submissions from the preprints deposited at an archive...and subjects them to peer review." (Suber 2004). A traditional journal serves five functions: registration, certification, awareness, archiving, rewarding (Casella \& Calvi 2010). A repository or preprint server performs all but one of these five functions - certification or peer review. An overlay journal performs the certification function. Combining the overlay journal and the preprint server addresses all five functions of a traditional journal.

Overlay journals were evaluated by addressing several key questions. Which preprint servers do overlay journals use? What licencing agreements facilitate the overlay journal? How does the preprint get considered by the overlay journal? Is peer review for the overlay journal similar to a traditional journal?

This paper aligns with the CAIS 2021 conference because it explores overlay journals, which can be considered overlooked since being created in the late 1990s and, at the same time, emergent to address an overwhelming number of preprints on the coronavirus disease 2019 (COVID-19).

\section{Methods}

Library and Information Science (LIS) Source (LISS) and LIS Abstracts (LISA) searches for "overlay journal" OR "overlay journals" returned 12 unique English language articles, and their bibliographies were screened. The same search terms were used in Google thereby including 
grey literature. For the first 10 pages of Google results, titles were screened. Purposive sampling was used in Google for software platforms and Google Scholar for one overlay journal title.

As a result of the proposed questions regarding overlay journals, 11 categories were created to analyze overlay journals' structure. The categories include "journal title," "year established as overlay," "first volume as overlay," "preprint server," "licence," "preprint identified by journal," "peer review," "software platform," "publisher," "support," and "associated fees." "Preprint identified by journal" indicates whether the author submits or the journal selects the articles. "Peer review" was populated with information regarding the reviewer's identity, recorded as either "identified" or "blinded," and the review's visibility, recorded as either "public" or "private." "Support" includes funding or administrative support information. Overlay journals were included if sufficient information was available, either from the journal's website or additional sources.

In addition to evaluating the overlay journal, the preprint server on which the journal overlays was analyzed. The number of preprints per year was investigated for three preprint servers: arXiv [1], bioRxiv [2], medRxiv [3]. For 2020, the number of COVID-19-related preprints [4] was compared to the total number of preprints. All analyses were performed in January 2021.

\section{Results}

The first overlay journals appeared in 1997 and overlaid on arXiv (Table 1). The arXiv preprint server launched in 1991 (Figure 1). With 300 preprints in 1991, arXiv had over 200,000 preprints deposited in 2020. BioRxiv started with only 70 preprints in 2013 and had 40,000 preprints deposited in 2020. Despite only launching in 2019 with 700 preprints, medRxiv had 14,000 preprints deposited in 2020 (Figure 1). For the year 2020, COVID-19 preprints represented 1\% of the total number of preprints in arXiv, 6\% in bioRxiv, and 61\% in medRxiv (Figure 2 ).

Table 1: Overlay Journal Characteristics

\begin{tabular}{|c|c|c|c|c|c|c|c|c|c|c|}
\hline Journal Title & \begin{tabular}{|l} 
Year \\
Established \\
as overlay
\end{tabular} & $\begin{array}{l}\text { First Volume } \\
\text { as overlay }\end{array}$ & $\begin{array}{l}\text { Preprint } \\
\text { Server }\end{array}$ & Licence & $\begin{array}{l}\text { Preprint } \\
\text { identified } \\
\text { by journal }\end{array}$ & $\begin{array}{l}\text { Peer } \\
\text { Review }\end{array}$ & \begin{tabular}{|l} 
Software \\
Platform
\end{tabular} & Publisher & Support & $\begin{array}{l}\text { Associated } \\
\text { Fees }\end{array}$ \\
\hline $\begin{array}{l}\text { Fundamenta } \\
\text { Informaticae [5] }\end{array}$ & $\begin{array}{l}2021 \\
\text { (switch to } \\
\text { overlay) }\end{array}$ & - & arXiv CoRR & & $\begin{array}{l}\text { Author } \\
\text { submitted }\end{array}$ & & Episciences & IOS Press & & Not stated \\
\hline $\begin{array}{l}\text { Rapid Reviews: } \\
\text { COVID19 } \\
\text { (RR:C19) [6] }\end{array}$ & 2020 & $\begin{array}{l}- \\
2020 \\
\text { Reviews } \\
\text { published; } \\
\text { no } \\
\text { Manuscripts } \\
\text { published }\end{array}$ & $\begin{array}{l}\text { medRxiv, } \\
\text { bioRxiv, } \\
\text { PsyArXiv, } \\
\text { SSRN, other } \\
\text { repositories } \\
\text { (not listed) }\end{array}$ & $\begin{array}{l}\text { CC BY } \\
4.0- \\
\text { Reviews }\end{array}$ & 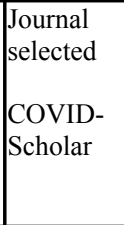 & $\begin{array}{l}\text { Identified/ } \\
\text { Public } \\
\text { Review has } \\
\text { DOI }\end{array}$ & $\begin{array}{l}\text { PubPub from } \\
\text { Knowledge } \\
\text { Futures } \\
\text { Group (MIT) }\end{array}$ & MIT Press & $\begin{array}{l}\text { Patrick J. } \\
\text { McGovern } \\
\text { Foundation }\end{array}$ & Not stated \\
\hline eLife [7] & \begin{tabular}{|l}
2020 \\
(switch to \\
overlay) \\
2021 \\
first \\
overlay \\
volume \\
to be \\
published
\end{tabular} & - & $\begin{array}{l}\text { bioRxiv, } \\
\text { medRxiv }\end{array}$ & $\begin{array}{l}\text { CC BY } \\
4.0\end{array}$ & $\begin{array}{l}\text { Author } \\
\text { submitted }\end{array}$ & $\begin{array}{l}\text { Identified/ } \\
\text { Public }\end{array}$ & & $\begin{array}{l}\text { eLife } \\
\text { Sciences } \\
\text { Publications, } \\
\text { Ltd. }\end{array}$ & $\begin{array}{l}\text { Howard } \\
\text { Hughes } \\
\text { Medical } \\
\text { Institute, the } \\
\text { Knut and } \\
\text { Alice } \\
\text { Wallenberg } \\
\text { Foundation, } \\
\text { the Max } \\
\text { Planck } \\
\text { Society, } \\
\text { Wellcome }\end{array}$ & $\begin{array}{l}\text { US } \$ 2500 \\
\text { author-paid } \\
\text { publication } \\
\text { fee }\end{array}$ \\
\hline
\end{tabular}




\begin{tabular}{|c|c|c|c|c|c|c|c|c|c|c|}
\hline $\begin{array}{l}\text { Journal of } \\
\text { Theoretical, } \\
\text { Computational } \\
\text { and Applied } \\
\text { Mechanics } \\
\text { (JTCAM) [8] }\end{array}$ & 2020 & - & arXiv, HAL & CC BY & $\begin{array}{l}\text { Author } \\
\text { submitted }\end{array}$ & $\begin{array}{l}\text { Blinded/ } \\
\text { Private; } \\
\text { Identified/ } \\
\text { Private }\end{array}$ & Episciences & & $\begin{array}{l}\text { Mecamat, } \\
\text { CCSD, } \\
\text { INRIA, } \\
\text { Loomio } \\
\text { Cooperative } \\
\text { Limited }\end{array}$ & $\begin{array}{l}\text { None for } \\
\text { author }\end{array}$ \\
\hline $\begin{array}{l}\text { Ars Inveniendi } \\
\text { Analytica [9] }\end{array}$ & 2020 & - & $\operatorname{arXiv}$ & $\begin{array}{l}\text { CC BY } \\
4.0\end{array}$ & $\begin{array}{l}\text { Author } \\
\text { submitted }\end{array}$ & $\begin{array}{l}\text { Blinded/ } \\
\text { Private }\end{array}$ & Scholastica & & & $\begin{array}{l}\text { None for } \\
\text { author; } \\
\text { US\$10 per } \\
\text { article** }\end{array}$ \\
\hline $\begin{array}{l}\text { Mathematical } \\
\text { Neuroscience } \\
\text { and Applications } \\
\text { (MNA) [10] }\end{array}$ & 2020 & - & $\operatorname{arXiv,~HAL~}$ & $\begin{array}{l}\text { CC BY } \\
4.0\end{array}$ & $\begin{array}{l}\text { Author } \\
\text { submitted }\end{array}$ & $\begin{array}{l}\text { Blinded/ } \\
\text { Private }\end{array}$ & Episciences & & & $\begin{array}{l}\text { None for } \\
\text { author }\end{array}$ \\
\hline ST-Open [11] & 2020 & 2020 & $\begin{array}{l}\text { University of } \\
\text { Split } \\
\text { repository, } \\
\text { DABAR, } \\
\text { other } \\
\text { Croatian } \\
\text { university } \\
\text { repositories }\end{array}$ & $\begin{array}{l}\text { CC BY } \\
4.0\end{array}$ & $\begin{array}{l}\text { Author } \\
\text { submitted; } \\
\text { Journal } \\
\text { selected }\end{array}$ & $\begin{array}{l}\text { Blinded/ } \\
\text { Private; } \\
\text { Identified/ } \\
\text { Private }\end{array}$ & OJS/PKP & $\begin{array}{l}\text { University of } \\
\text { Split }\end{array}$ & & $\begin{array}{l}\text { None for } \\
\text { author }\end{array}$ \\
\hline $\begin{array}{l}\text { Machine } \\
\text { Learning for } \\
\text { Biomedical } \\
\text { Imaging } \\
\text { (MELBA) } \\
\text { Journal [12] }\end{array}$ & 2020 & 2020 & $\operatorname{arXiv}$ & $\begin{array}{l}\text { CC } \\
\text { licencing }\end{array}$ & $\begin{array}{l}\text { Author } \\
\text { submitted }\end{array}$ & $\begin{array}{l}\text { Blinded/ } \\
\text { Private }\end{array}$ & Scholastica & & & $\begin{array}{l}\text { Not stated; } \\
\text { US\$10 per } \\
\text { article*** }\end{array}$ \\
\hline $\begin{array}{l}\text { Épijournal de } \\
\text { Didactique et } \\
\text { Epistémologie } \\
\text { des } \\
\text { Mathématiques } \\
\text { pour } \\
\text { l'Enseignement } \\
\text { Supérieur } \\
\text { (EpiDEMES) } \\
{[13]}\end{array}$ & 2019 & - & $\operatorname{arXiv,~HAL~}$ & $\begin{array}{l}\text { CC BY - } \\
\text { SA }\end{array}$ & $\begin{array}{l}\text { Author } \\
\text { submitted }\end{array}$ & $\begin{array}{l}\text { Blinded/ } \\
\text { Private }\end{array}$ & Episciences & & CCSD & $\begin{array}{l}\text { None for } \\
\text { author }\end{array}$ \\
\hline $\begin{array}{l}\text { Advances in } \\
\text { Combinatorics } \\
{[14]}\end{array}$ & 2019 & 2019 & $\operatorname{arXiv}$ & $\mathrm{CC} \mathrm{BY}$ & $\begin{array}{l}\text { Author } \\
\text { submitted }\end{array}$ & $\begin{array}{l}\text { Blinded/ } \\
\text { Private }\end{array}$ & Scholastica & \begin{tabular}{|l} 
Alliance of \\
Diamond \\
Open Access \\
Journals
\end{tabular} & \begin{tabular}{|l} 
Queen's \\
University \\
Library
\end{tabular} & $\begin{array}{l}\text { None for } \\
\text { author; } \\
\text { US\$10 per } \\
\text { article** }\end{array}$ \\
\hline $\begin{array}{l}\text { Journal of } \\
\text { Nonsmooth } \\
\text { Analysis and } \\
\text { ptimization } \\
\text { (JNSAO) [15] }\end{array}$ & 2019 & 2020 & $\operatorname{arXiv,~HAL~}$ & $\begin{array}{l}\mathrm{CC} \\
\text { licencing }\end{array}$ & $\begin{array}{l}\text { Author } \\
\text { submitted }\end{array}$ & $\begin{array}{l}\text { Blinded/ } \\
\text { Private }\end{array}$ & Episciences & \begin{tabular}{|l} 
JNSAO \\
Editorial \\
Board
\end{tabular} & & $\begin{array}{l}\text { None for } \\
\text { author }\end{array}$ \\
\hline JMIRx Med [16] & 2019 & 2020 & medRxiv & $\begin{array}{l}\text { CC BY - } \\
\text { articles } \\
\text { and } \\
\text { reviews; } \\
\text { CC0 - } \\
\text { images }\end{array}$ & $\begin{array}{l}\text { Author } \\
\text { submitted; } \\
\text { Journal } \\
\text { selected }\end{array}$ & $\begin{array}{l}\text { Identified/ } \\
\text { Public } \\
\text { Review has } \\
\text { DOI }\end{array}$ & & JMIR & & $\begin{array}{l}\text { None for } \\
\text { author }\end{array}$ \\
\hline JMIRx Bio [17] & 2019 & - & bioRxiv & $\begin{array}{l}\text { CC BY - } \\
\text { articles } \\
\text { and } \\
\text { reviews; } \\
\text { CC0 - } \\
\text { images }\end{array}$ & $\begin{array}{l}\text { Author } \\
\text { submitted; } \\
\text { Journal } \\
\text { selected }\end{array}$ & $\begin{array}{l}\text { Identified/ } \\
\text { Public } \\
\text { Review has } \\
\text { DOI }\end{array}$ & & JMIR & & $\begin{array}{l}\text { None for } \\
\text { author }\end{array}$ \\
\hline
\end{tabular}




\begin{tabular}{|c|c|c|c|c|c|c|c|c|c|c|}
\hline JMIRx Psy [17] & 2019 & T- & PsyArXiv & $\begin{array}{l}\text { CC BY - } \\
\text { articles } \\
\text { and } \\
\text { reviews; } \\
\text { CC0 - } \\
\text { images } \\
\end{array}$ & $\begin{array}{l}\text { Author } \\
\text { submitted; } \\
\text { Journal } \\
\text { selected }\end{array}$ & $\begin{array}{l}\text { Identified/ } \\
\text { Public } \\
\text { Review has } \\
\text { DOI }\end{array}$ & & JMIR & & $\begin{array}{l}\text { None for } \\
\text { author }\end{array}$ \\
\hline $\begin{array}{l}\text { Neurons, } \\
\text { Behavior, Data } \\
\text { analysis and } \\
\text { Theory (NBDT) } \\
{[18]}\end{array}$ & 2018 & 2018 & $\operatorname{arXiv}$ & $\mathrm{CC} \mathrm{BY}$ & $\begin{array}{l}\text { Author } \\
\text { submitted }\end{array}$ & $\begin{array}{l}\text { Blinded/ } \\
\text { Private; } \\
\text { Blinded/ } \\
\text { Public }\end{array}$ & Scholastica & $\begin{array}{l}\text { The neurons, } \\
\text { behavior, } \\
\text { data analysis } \\
\text { and theory } \\
\text { collective }\end{array}$ & & $\begin{array}{l}\text { None for } \\
\text { author; } \\
\text { US\$10 per } \\
\text { article** }\end{array}$ \\
\hline biOverlay [19] & $\begin{array}{l}2018 \\
2020 \\
\text { closed }\end{array}$ & 2018 & not listed & $\begin{array}{l}\text { CC BY - } \\
\text { reviews }\end{array}$ & $\begin{array}{l}\text { Journal } \\
\text { selected }\end{array}$ & $\begin{array}{l}\text { Blinded/ } \\
\text { Public; } \\
\text { Identified/ } \\
\text { Public }\end{array}$ & & & \begin{tabular}{|l} 
Gordon and \\
Betty Moore \\
Foundation
\end{tabular} & \\
\hline The Idealis* [20 & $\begin{array}{l}2017 \\
2019[21] \\
\text { closed }\end{array}$ & 2017 & & & $\begin{array}{l}\text { Journal } \\
\text { selected }\end{array}$ & & $\begin{array}{l}\text { WordPress; } \\
\text { Press- } \\
\text { Forward }\end{array}$ & & & \\
\hline Quantum [22] & 2017 & 2017 & $\operatorname{arXiv}$ & $\begin{array}{l}\text { CC BY } \\
4.0\end{array}$ & $\begin{array}{l}\text { Author } \\
\text { submitted }\end{array}$ & $\begin{array}{l}\text { Blinded/ } \\
\text { Private }\end{array}$ & Scholastica & & & $\begin{array}{l}\text { Author paid } \\
\text { publication } \\
\text { fees: regular } \\
€ 450, \\
\text { discounted } \\
€ 100 \text {, or } \\
\text { waived } € 0 ; \\
\text { Quantum } \\
\text { pays US } \$ 10 \\
\text { per article** }\end{array}$ \\
\hline $\begin{array}{l}\text { Épijournal de } \\
\text { Géométrie } \\
\text { Algébrique } \\
\text { (Epiga) [23] }\end{array}$ & 2016 & 2017 & arXiv, HAL & $\begin{array}{l}\text { CC BY - } \\
\text { SA }\end{array}$ & $\begin{array}{l}\text { Author } \\
\text { submitted }\end{array}$ & $\begin{array}{l}\text { Blinded/ } \\
\text { Private }\end{array}$ & Episciences & & CCSD & $\begin{array}{l}\text { None for } \\
\text { author }\end{array}$ \\
\hline \begin{tabular}{|l} 
Discrete \\
Analysis [24]
\end{tabular} & 2016 & 2016 & $\operatorname{arXiv}$ & $\mathrm{CC} \mathrm{BY}$ & $\begin{array}{l}\text { Author } \\
\text { submitted }\end{array}$ & \begin{tabular}{|l} 
Blinded/ \\
Private
\end{tabular} & Scholastica & \begin{tabular}{|l|} 
Alliance of \\
Diamond \\
Open Access \\
Journals \\
\end{tabular} & \begin{tabular}{|l} 
Cambridge \\
University \\
and Stanhill \\
Foundation \\
\end{tabular} & $\begin{array}{l}\text { None for } \\
\text { author; } \\
\text { US\$10 per } \\
\text { article** }\end{array}$ \\
\hline $\begin{array}{l}\text { The Open } \\
\text { Journal of } \\
\text { Astrophysics } \\
{[25]} \\
\end{array}$ & 2016 & 2016 & $\operatorname{arXiv}$ & CC BY & $\begin{array}{l}\text { Author } \\
\text { submitted }\end{array}$ & $\begin{array}{l}\text { Blinded/ } \\
\text { Private }\end{array}$ & Scholastica & $\begin{array}{l}\text { Maynooth } \\
\text { Academic } \\
\text { Publishing }\end{array}$ & \begin{tabular}{|l} 
Gordon and \\
Betty Moore \\
Foundation
\end{tabular} & $\begin{array}{l}\text { None for } \\
\text { author; } \\
\text { US\$10 per } \\
\text { article** }\end{array}$ \\
\hline $\begin{array}{l}\text { Journal of Inter- } \\
\text { disciplinary } \\
\text { Methodologies } \\
\text { and Issues in } \\
\text { Science (JIMIS) } \\
\text { [26] }\end{array}$ & 2016 & 2016 & arXiv, HAL & $\begin{array}{l}\text { CC } \\
\text { licencing }\end{array}$ & $\begin{array}{l}\text { Author } \\
\text { submitted }\end{array}$ & $\begin{array}{l}\text { Blinded/ } \\
\text { Private }\end{array}$ & Episciences & & \begin{tabular}{|l} 
UMR \\
ESPACE 7300 \\
(CNRS, \\
Université \\
d'Avignon et \\
des Pays de \\
Vaucluse), \\
CCSD, (S)FR \\
Agorantic
\end{tabular} & Not stated \\
\hline $\begin{array}{l}\text { Hardy-Ramanu- } \\
\text { jan Journal [27] }\end{array}$ & $\begin{array}{l}2014 \\
\text { (switch to } \\
\text { overlay) }\end{array}$ & 2015 & arXiv, HAL & $\begin{array}{l}\text { CC } \\
\text { licencing }\end{array}$ & $\begin{array}{l}\text { Author } \\
\text { submitted }\end{array}$ & $\begin{array}{l}\text { Blinded/ } \\
\text { Private }\end{array}$ & Episciences & & & Not stated \\
\hline $\begin{array}{l}\text { Discrete } \\
\text { Mathematics \& } \\
\text { Theoretical } \\
\text { Computer } \\
\text { Science } \\
\text { (DMTCS) [28] }\end{array}$ & $\begin{array}{l}2014 * * * \\
\text { (switch to } \\
\text { overlay) }\end{array}$ & 2015 & arXiv, HAL & $\begin{array}{l}\text { CC BY } \\
4.0\end{array}$ & $\begin{array}{l}\text { Author } \\
\text { submitted }\end{array}$ & \begin{tabular}{|l} 
Blinded/ \\
Private
\end{tabular} & Episciences & \begin{tabular}{|l} 
Discrete \\
Mathematics \\
and \\
Theoretical \\
Computer \\
Science \\
(DMTCS) \\
\end{tabular} & Inria & $\begin{array}{l}\text { None for } \\
\text { author }\end{array}$ \\
\hline $\begin{array}{l}\text { Journal of Data } \\
\text { Mining and } \\
\text { Digital } \\
\text { Humanities } \\
\text { (JDMDH) [29] }\end{array}$ & 2014 & 2014 & $\begin{array}{l}\text { arXiv, HAL, } \\
\text { CWI, } \\
\text { PRODINRA }\end{array}$ & $\begin{array}{l}\text { CC BY } \\
4.0\end{array}$ & $\begin{array}{l}\text { Author } \\
\text { submitted }\end{array}$ & $\begin{array}{l}\text { Blinded/ } \\
\text { Private }\end{array}$ & Episciences & & $\begin{array}{l}\text { INRA, Inria, } \\
\text { CNRS }\end{array}$ & $\begin{array}{l}\text { None for } \\
\text { author }\end{array}$ \\
\hline
\end{tabular}




\begin{tabular}{|c|c|c|c|c|c|c|c|c|c|c|}
\hline $\begin{array}{l}\text { (JIPS) Journal } \\
\text { d'Interaction } \\
\text { Homme } \\
\text { Machines [30] }\end{array}$ & 2008 & 2010 & $\begin{array}{l}\text { arXiv, HAL, } \\
\text { CWI }\end{array}$ & $\begin{array}{l}\text { CC BY } \\
2.0\end{array}$ & \begin{tabular}{|l} 
Author \\
submitted
\end{tabular} & $\begin{array}{l}\text { Blinded/ } \\
\text { Private }\end{array}$ & $\begin{array}{l}\text { Episciences } \\
\text { as of } \\
2014^{* * *}\end{array}$ & \begin{tabular}{|l|} 
Franco- \\
phone \\
Association \\
for Human- \\
Computer \\
Interaction \\
(AFIHM)
\end{tabular} & & $\begin{array}{l}\text { None for } \\
\text { author }\end{array}$ \\
\hline $\begin{array}{l}\text { Symmetry, } \\
\text { Integrability and } \\
\text { Geometry: } \\
\text { Methods and } \\
\text { Applications } \\
\text { (SIGMA) [31] }\end{array}$ & 2005 & 2005 & arXiv & $\begin{array}{l}\text { CC BY - } \\
\text { SA }\end{array}$ & $\begin{array}{l}\text { Author } \\
\text { submitted }\end{array}$ & \begin{tabular}{|l|} 
Blinded/ \\
Private
\end{tabular} & & & \begin{tabular}{|l} 
Foundation \\
Compositio \\
Mathematica, \\
University \\
Library of \\
Radboud \\
University \\
Nijimegen, \\
Sociedad \\
Mexicana de \\
Física, \\
University \\
Libraries of the \\
Delft \\
University of \\
Technology \\
and Uppsala \\
University \\
\end{tabular} & $\begin{array}{l}\text { None for } \\
\text { author }\end{array}$ \\
\hline \begin{tabular}{l|} 
Logical Methods \\
in Computer \\
Science (LMCS) \\
[32]
\end{tabular} & 2004 & 2005 & arXiv CoRR & \begin{tabular}{|l|}
$\mathrm{CC}$ \\
licencing
\end{tabular} & $\begin{array}{l}\text { Author } \\
\text { submitted }\end{array}$ & $\begin{array}{l}\text { Blinded/ } \\
\text { Private }\end{array}$ & \begin{tabular}{|l|} 
Episciences \\
as of \\
$2014^{* * *}$
\end{tabular} & $\begin{array}{l}\text { Logical } \\
\text { Methods in } \\
\text { Computer } \\
\text { Science e.V. }\end{array}$ & & $\begin{array}{l}\text { None for } \\
\text { author }\end{array}$ \\
\hline $\begin{array}{l}\text { African Journal } \\
\text { of Research in } \\
\text { Computer } \\
\text { Science and } \\
\text { Applied } \\
\text { Mathematics } \\
\text { (ARIMA) } \\
\text { Journal [33] }\end{array}$ & 2002 & 2002 & HAL & \begin{tabular}{|l|}
$\mathrm{CC}$ \\
licencing
\end{tabular} & $\begin{array}{l}\text { Author } \\
\text { submitted }\end{array}$ & $\begin{array}{l}\text { Blinded/ } \\
\text { Private }\end{array}$ & $\begin{array}{l}\text { Episciences } \\
\text { as of } \\
2014 * * *\end{array}$ & & & Not stated \\
\hline $\begin{array}{l}\text { Geometry and } \\
\text { Topology } \\
\text { (G\&T) [34] }\end{array}$ & $\begin{array}{l}1997 \\
2012^{* * * * *} \\
\text { no longer } \\
\text { overlay }\end{array}$ & $\begin{array}{l}1997 \\
1997-2007 \\
\text { on arXiv }\end{array}$ & arXiv & \begin{tabular}{|l|}
$1997-2005$ \\
copyright \\
Geometry \\
$\&$ \\
Topology \\
Publica- \\
tions \\
2006-2007 \\
no \\
copyright \\
statement
\end{tabular} & $\begin{array}{l}\text { Author } \\
\text { submitted }\end{array}$ & $\begin{array}{l}\text { Blinded/ } \\
\text { Private }\end{array}$ & & & & \\
\hline \begin{tabular}{|l|} 
Journal of High \\
Energy Physics \\
(JHEP) [35]
\end{tabular} & $\begin{array}{l}1997 \\
2012^{* * * * *} \\
\text { no longer } \\
\text { overlay }\end{array}$ & 1997 & arXiv & \begin{tabular}{|l|}
$1997-2002$ \\
no \\
copyright \\
statement \\
$2002-2006$ \\
copyright \\
SISA A \\
ISAS \\
$2007-2009$ \\
lopyright \\
SISSA
\end{tabular} & $\begin{array}{l}\text { Author } \\
\text { submitted }\end{array}$ & \begin{tabular}{|l|} 
Blinded/ \\
Private
\end{tabular} & $\begin{array}{l}\text { developed by } \\
\text { SISSA }\end{array}$ & $\begin{array}{l}\text { 2002-2009 } \\
\text { Institute of } \\
\text { Physics } \\
\text { (IOP) } \\
\text { Publishing }\end{array}$ & & \\
\hline \multicolumn{11}{|c|}{$\begin{array}{l}\text { *Overlay no longer functioning and the website cannot be accessed. Information is from (Troia 2017). } \\
* * \text { (Conover 2016; Ball 2015) } \\
* * * \text { (Berthaud et al. 2014) } \\
* * * * \text { (Priem \& Hemminger 2012) }\end{array}$} \\
\hline
\end{tabular}




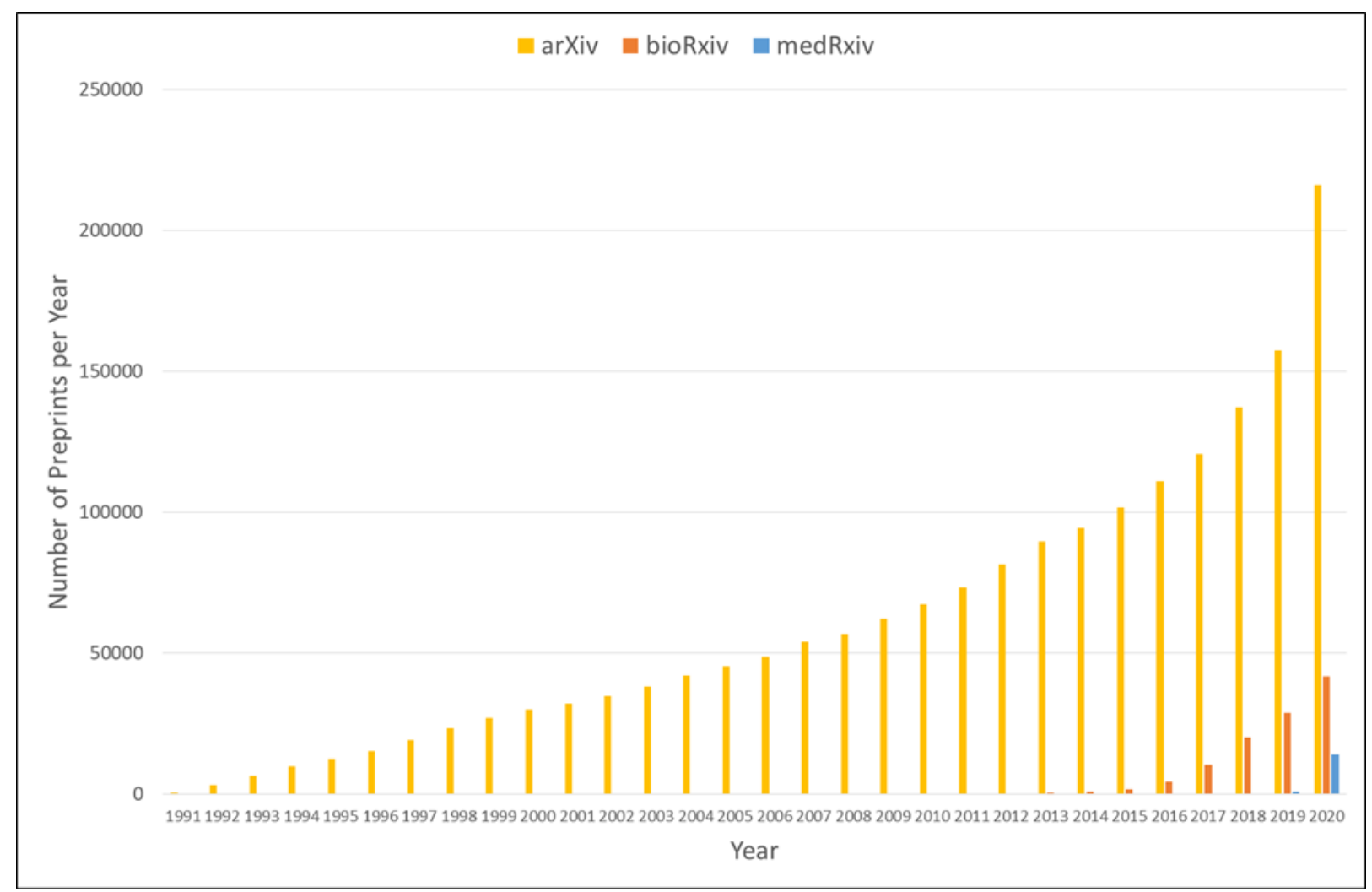

Figure 1: Number of Preprints per Year in arXiv (1991-); bioRxiv (2013-); medRxiv (2019-)

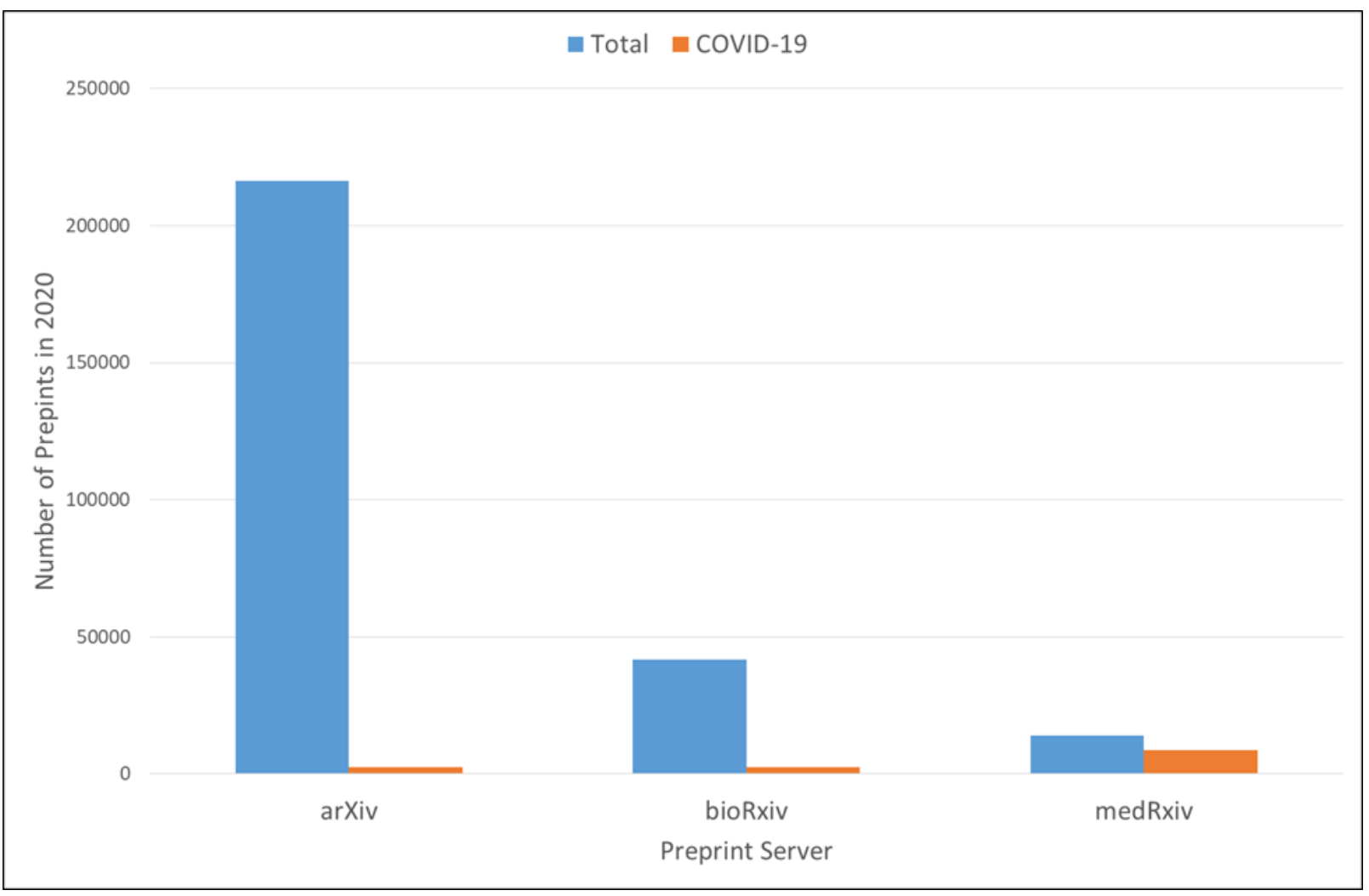

Figure 2: Total and COVID-19 Preprints in 2020 in arXiv (1\%); bioRxiv (6\%); medRxiv (61\%) 
Thirty-one overlay journals with sufficient information were identified (Table 1). Twenty-two published at least one volume of the overlay journal, and nine were established but have not published their first volume of journal articles as an overlay journal (Table 1). Of the 22 published overlay journals, four are no longer overlays. Priem \& Henninger (2012) report that Geometry and Topology (G\&T) and Journal of High Energy (JHEP), both established in 1997, have ceased to be overlay journals. The Idealis, a LIS overlay journal, spanned from 2017 (Troia, 2017) to 2019 [21]. Unfortunately, the website for The Idealis has been suspended [20]. Additionally, biOverlay was designed as an "experiment with the goal of testing detailed, portable comments for preprints" (Greene 2020) and spanned from 2018-2020. Established in 2020, RR:C19 has published peer reviews but has not published a formal manuscript volume yet. While eLife started in 2012, it is in the process of switching to an overlay between December 2020 and July 2021 (Eisen et al. 2020). Likewise, two of the 23 published overlay journals switched to an overlay from a traditional publishing model (Table 1). Berthaud (2014) reports that Discrete Mathematics \& Theoretical Computer Science (DMTCS) was in the process of switching to an overlay using Episciences software.

Twenty-two of 31 overlays use arXiv as a preprint server (Table 1). After 2019, four overlays use medRxiv and/or bioRxiv (Figure 1). Some journals use one server: PsyArXiv or Hyper Articles en Ligne (HAL). ST-Open uses Croatian repositories as its purpose is to promote Croatian students' research visibility and quality (Marusic et al. 2019).

Where licencing and copyright information was given, most of the overlays required Creative Commons (CC) licencing of some sort, usually as determined by the preprint server (Table 1). The first set of CC licences were available in 2002 (Creative Commons n.d.). Two overlays started in 1997. $G \& T$ copyrighted the arXiv version using Geometry and Topology Publications from 1997-2005 and provided no copyright statement from 2006-2007. JHEP provided no copyright statement from 1997-2002, copyright SISSA/ISAS (Scuola Internazionale Superiore di Studi Avanzati/International School for Advanced Studies) from 2002-2006, and copyright SISSA from 2007-2009.

Twenty-eight of 31 overlays include author submitted content; however, seven overlays seek out preprints. ST-Open uses a hybrid method in which authors can submit preprints or editors can select, solicit, or recommend preprints to the overlay journal. JMIRx overlays use acquisition and review editors with the option for authors to self-nominate. $R R: C 19$ identifies preprints for review using COVIDScholar (University of California, Berkeley n.d.).

Twenty-three overlays use a blinded and private peer review process. Starting in 2018, RR:C19, JMIRx overlays, eLife, and biOverlay provide identified and public peer reviews. RR:C19 and JMIRx Med publish peer reviews with a digital object identifier (DOI). Journal of Theoretical, Computational and Applied Mechanics (JTCAM) provides the option for blinded or identified private reviews. Neurons, Behavior, Data analysis and Theory (NBDT) reviews are public or private, blinded reviews.

Episciences (starting in 2014) and Scholastica (starting in 2016) are the dominant software platforms (Table 1). Thirteen overlays use Episcience, a free software (Episciences, n.d.), and 
seven overlays use Scholastica. Some overlays have custom-developed software, such as PubPub from Knowledge Futures Group for RR:C19. Other options include Open Journal System by Public Knowledge Project and WordPress with PressForward.

The least consistent details regarding overlay journals are publisher, support and fees (Table 1). Sixteen overlays listed a publisher, while 15 overlays did not. In some cases, the publisher is a discipline-specific association. While 19 overlays do not charge authors, eLife charges US\$2500 and Quantum charges $€ 450$, which can be discounted or waived. However, costs are associated with the production of overlay journals. Scholastica charges the journal US\$10 per published article (Conover 2016; Ball 2015). As such, a wide variety of funding models exist, with donors, institutions (government organizations, libraries, and universities), and foundations covering fees. For example, Queen's University Library covers Advances in Combinatorics' costs while also offering administrative support (Queen's Gazette, 2018).

\section{Discussion}

Overlay journals can be divided into two groups: historically conventional, overlooked journals and new, emergent journals. Conventional overlay journals use arXiv, include articles submitted by authors via preprint servers, and provide blinded and private peer review. Recently, emergent overlay journals use bioRxiv or medRxiv, include articles found by the journal on preprint servers, and provide identified and public peer review.

The motivation for overlay journals has been directly or indirectly attributed to costs of traditional journals (Neumann 2010; Ball 2015; Conover 2016) or directly related to the volume of preprints in the current pandemic (Free 2020) (Figure 2). Overlay journals provide certification to an otherwise uncertified work, ensuring the accuracy of information in a timely fashion. Some journals switched from overlay to traditional because they were not self-sustaining, even with donations of time and funds (Neumann 2010; Fosmire 2013). The effect of newer software platforms on long-term sustainability is yet to be determined. Overlay journals are a relatively inexpensive way to certify and publish material.

From an information literacy perspective, information professionals and researchers need to be aware of potentially unintended consequences. One concern is that, as journals select preprints, different versions of the same preprint could appear in multiple journals (Vines 2019; Lab 2020). How can an author prevent their work from an "unauthorized" journal reviewing it without the author's "consent?" How is it decided who gets the first pick for an article? However, one could also argue that a researcher implicitly gives up the ability to gatekeep access to his/her article when posting a preprint on an archive.

With organizations, such as the Confederation of Open Access Repositories (COAR), arguing for overlays as the "model of the future" (COAR n.d.), it is important to be aware of the consequences of overlays, their emerging trends, and the opportunities for improved scholarly communication. Overlay journals allow community and collegial feedback, and collaboration across disciplines at a reasonable price (currently). For example, mathematics and physics have created an atmosphere of collegial sharing and critique using preprints and current research (Berthaud 2014, 275; Herman 2020, 218). Similarly, NBDT selects works based on whether the 
editor would consider running a preprint as a "journal club paper for their own lab" (NBDT collective n.d.). Herman (2020) also argues that "there is an increasing scope for a number of new overlay journals to be developed, tailored for different research communities" (218).

In concluding, the use of overlay journals has implications, certainly for scholarly communications, but also for researchers and library and information professionals. Overlay journals, as a means of scholarly communication, have served to further highlight the importance of and continued need for peer review as overlay journals have become a way to meet the fast-paced and quickly evolving information needs of individuals today, especially during a global pandemic. For researchers, overlay journals can potentially provide increased discoverability earlier in the journal publication process; however, this increased discoverability and openness could have potential consequences if authors are looking to publish in different journals but a journal-selected overlay has already reviewed or "claimed" the preprint. Overlay journals can also potentially offer researchers a way to meet grant funder open access requirements and, in doing so, also provide a cost effective publishing option. For library and information professionals, overlay journals, in adding the certification to otherwise uncertified preprints, are a helpful way to evaluate the authority and legitimacy of rapidly developing information. As well, overlay journals are an avenue for identifying timely, relevant, and sought after information to support the information needs of information seekers in a time of growing information. Ultimately, whether overlooked or emergent, overlay journals appear to be here to stay.

\section{URL List:}

1. arXiv advanced search: https://arxiv.org/search/advanced

2. bioRxiv advanced search: https://www.biorxiv.org/search

3. medRxiv advanced search: https://www.medrxiv.org/search

4. Covid-19 preprint search: https://icite.od.nih.gov/covid19/search/

5. Fundamenta Informaticae: https://fi.episciences.org/

6. RR:C19: https://rapidreviewscovid19.mitpress.mit.edu/

7. eLife: $\underline{\text { https://elifesciences.org/ }}$

8. JTCAM: https://itcam.episciences.org/

9. Ars Inveniendi Analytica: https://ars-inveniendi-analytica.com/

10. $M N A$ : https://mna.episciences.org/

11. ST-Open: http://st-open.unist.hr/index.php/st-open

12. MELBA: https://www.melba-journal.org/

13. epiDEMES: https://epidemes.episciences.org/

14. Advances in Combinatorics: https://www.advancesincombinatorics.com/

15. JNSAO: https://jnsao.episciences.org/

16. JMIRx Med: https://xmed.jmir.org/

17. JMIRx: https://support.jmir.org/hc/en-us/articles/360034752692

18. NBDT: https://nbdt.scholasticahq.com/

19. biOverlay: https://www.bioverlay.org/

20. The Idealis: www.theidealis.org 
21. Google Scholar "The Idealis" search:

https://scholar.google.com/scholar?hl=en\&as $\mathrm{sdt}=0,5 \& \mathrm{q}=$ "The+Idealis"

22. Quantum: https://quantum-journal.org/

23. Epiga: https://epiga.episciences.org/page/a-propos

24. Discrete Analysis: https://discreteanalysisjournal.com/

25. The Open Journal of Astrophysics: https://astro.theoj.org/

26. JIMIS: https://jimis.episciences.org/

27. Hardy Ramanujan Journal: https://hri.episciences.org/

28. DTMCS: https://dmtcs.episciences.org/

29. JDMDH: https://idmdh.episciences.org/

30. JIPS: https://jips.episciences.org/

31. SIGMA: https://www.emis.de/journals/SIGMA/

32. LMCS: https://lmcs.episciences.org/

33. ARIMA: https://arima.episciences.org/

34. G\&T: https://msp.org/gt/about/journal/about.html

35. JHEP: https://jhep.sissa.it/jhep/index.jsp

\section{Reference List:}

Ball, P. (2015). The journal that publishes no papers: Mathematics journal 'overlays' arXiv preprint server. Nature, 526, 146.

Berthaud, C., Capelli, L., Gustedt, J., Kirchner, C., Loiseau, K., Magron, A., Medves, M., Monteil, A., Rivrieux, G., \& Romary, L. (2014). EPISCIENCES - An overlay publication platform: Let's Put Data to Use: Digital Scholarship for the Next Generation. Information Services \& Use, 34(3-4), 269-277. doi:10.3233/ISU-140749

Brown, J. (2010). An introduction to overlay journals. Repositories Support Project: UK. https://discovery.ucl.ac.uk/id/eprint/19081/

Cassella, M., \& Calvi, L. (2010). New journal models and publishing perspectives in the evolving digital environment. IFLA Journal, 36(1), 7-15. doi:10.1177/0340035209359559

Confederation of Open Access Repositories (COAR). (n.d.). Is overlay the peer review the future of scholarly communications? Confederation of Open Access Repositories. https://www.coar-repositories.org/news-updates/is-overlay-peer-review-the-future-of-sch olarly-communications/

Conover, E. (2016, February). New journals piggyback on arXiv. APS News 25(2).

Creative Commons. (n.d.). 1.1 The Story of Creative Commons. Creative Commons Certificate for Educators and Librarians.

https://certificates.creativecommons.org/cccertedu/chapter/1-1-the-story-of-creative-com mons/

Eisen, M. B., Akhmanova, A., Behrens, T. E., Harper, D. M., Weigel, D., \& Zaidi, M. (2020). Implementing a "publish, then review" model of publishing. eLife, 9, e64910. doi:10.7554/eLife. 64910

Episciences. (n.d.). Overlay journal platform. Episciences.org. https://www.episciences.org/

Fosmire, M. (2013). The Sudden Selector's Guide to Physics Resources. Libraries Faculty and Staff Scholarship and Research. Paper 57. http://docs.lib.purdue.edu/lib_fsdocs/57 
Free, D. (2020). The MIT Press launches Rapid Reviews: COVID-19. College \& Research Libraries News, 81(8), 373. doi:10.5860/crln.81.8.370

Greene, C. (2020). biOverlay experiment summary. biOverlay. https:/www.bioverlay.org/post/2020-01-experiment-summary/

Herman, E., Akeroyd, J., Bequet, G., Nicholas, D., \& Watkinson, A. (2020). The changed - and changing - landscape of serials publishing: Review of the literature on emerging models. Learned Publishing, 33, 213-229. doi:10.1002/leap.1288

Lab, R. [@RaslerLab]. (2020, Dec 19)._Hi friends at@ASAPbio_and fans or preprints, I really need your help. I'm a bit fan of \#preprints, but there is new development that if it finds copycats,will totallykill preprintsat@biorxivand@medrxiv.There is a journal at @mitpress, called 'Rapid REviews COVID19'. It.. [tweet thread]. Twitter. https://twitter.com/RalserLab/status/1340284808224792578

Marušić, M., Tomić, V., Gudelj, D., Wager, E., \& Marušić, A. (2019). University repository overlay journal - increasing the quality and visibility of student research at the University of Split, Croatia. European Science Editing, 45(2), 39-41. doi:10.20316/ESE.2019.45.19007

Neumann, W. (2010). Walter Neumann on the Success of Geometry \& Topology. ScienceWatch.com. http://archive.sciencewatch.com/inter/jou/pdf/10mayGeoTop.pdf

Neuron, Behaviour, Data Analysis, and Theory (NBDT) collective. (n.d.). For authors. NBDT. https://nbdt.scholasticahq.com/for-authors

Priem, J. \& Hemminger, B. (2012). Decoupling the scholarly journal. Frontiers in Computational Neuroscience 6(19), 1-13. doi:10.3389/fncom.2012.00019

Queen's Gazette (2018, June 12) Queen's University Library collaborates on innovative journal. Retrieved from https:/www.queensu.ca/gazette/stories/queen-s-university-library-publishes-new-journaladvances-combinatorics

Suber, P. (2004, July 14). Guide to the Open Access Movement. http://legacy.earlham.edu/ peters/fos/guide.htm

Troia, L. (2017). Leading by example: The Idealis highlights expert-curated open access LIS research. ARClog. https://acrlog.org/2017/03/29/leading-by-example-the-idealis-highlights-expert-curated-o pen-access-lis-research/\#more-6759

Vines, T. (2019, Aug 15). Two new initiatives at eLife to start the Eisen era. Scholarly Kitchen. https://scholarlykitchen.sspnet.org/2019/08/15/two-new-initiatives-at-elife-to-start-the-eis en-era/

University of California, Berkeley. (n.d.). Berkeley Public Health's Stefano Bertozzi Is Editor-in-Chief of New Peer-Reviewed COVID-19 Journal. Berkeley Public Health. https://publichealth.berkeley.edu/news-media/faculty-honors/stefano-bertozzi-is-editor-in -chief-of-new-peer-reviewed-covid-19-journal/ 